\title{
Modelling and verification of melanin concentration on human skin type
}

\author{
Mrs Aletta E Karsten ${ }^{1,2}$, Dr Jacoba E Smit*,3,4
}

${ }^{1}$ Biophotonics group, National Laser Centre, CSIR, PO Box 395, Pretoria, 0001, South Africa

${ }^{2}$ Department of Physics, University of Pretoria, Pretoria, 0002, South Africa

${ }^{3}$ Biosciences, CSIR, PO Box 395, Pretoria, 0001, South Africa

${ }^{4}$ Modelling and Digital Sciences, CSIR, PO Box 395, Pretoria, 0001, South Africa

*Corresponding author's name and email: Jacoba E Smit (KSmit@csir.co.za)

\footnotetext{
${ }^{*}$ To whom correspondence should be addressed, at Modelling and Digital Sciences, CSIR, PO Box 395, Pretoria, 0001, South Africa; fax: + 2712841 2456; email: KSmit@csir.co.za;
} 


\section{ABSTRACT}

Lasers are used in the minimalistic or non-invasive diagnosis and treatment of skin disorders. Less laser light reaches the deeper skin layers in dark skin types, due to its higher epidermal melanin concentration compared to lighter skin. Laser-tissue interaction modelling software can correct for this by adapting the dose applied to the skin. This necessitates an easy and reliable method to determine the skin's type. Non-invasive measurement of the skin's melanin content is the best method. However, access to samples of all skin types is often limited and skin-like phantoms are used instead. This study's objective is to compare experimentally measured absorption features of liquid skin-like phantoms representing Skin Types I to VI with a realistic skin computational model component of ASAP®. Sample UVVIS transmittance spectra were measured from 370 to $900 \mathrm{~nm}$ and compared to simulated results from ASAP® using the same optical parameters. Results indicated non-monotonic absorption features towards shorter wavelengths, which may allow for more accurate ways of determining melanin concentration and expected absorption through the epidermal layer. This suggests possible use in representing optical characteristics of real skin. However, a more comprehensive model and phantoms are necessary to account for the effects of sun exposure. 


\section{INTRODUCTION}

In the past 50 years lasers have found numerous applications in medicine, both for treatment and diagnostics (1). One of the advantages of lasers is that they can be used for minimalistic or non-invasive diagnosis and treatment. Often that means that the light must penetrate through the skin and the correct dose required, relies on accurate information regarding the skin's optical properties (2).

Human skin consists of different layers, with the epidermal layer containing the melanin that is responsible for skin tone or skin type (3). Epidermal melanin can broadly be divided into two types, eumelanin (black-brown colour) and pheomelanin (yellow-reddish colour) $(3,4)$. Melanin is synthesized within melanosomes inside melanocytes located in the basal layer of the epidermis and the mature melanosomes get transferred via dendrites to the keratinocytes in die epidermis where they are responsible for skin photoprotection (3).

It is well documented that the absorption and scattering of light through skin tissue depends on the skin's optical properties (see for example studies by Tuchin (5)). Melanin absorbs light in the visible and near infrared parts of the spectrum, the part most suitable for diagnostics and treatment due to deeper penetration (1). Absorption of light in the epidermal layer is a major factor in determining the fluence of the laser light that reaches the deeper levels of the skin. Darker skin has an epidermal melanin volume fraction of about twice that of lighter skins (6). Due to melanin absorption, less laser light reaches the deeper parts of the skin in dark skin types (1). This can be corrected for by laser-tissue interaction modelling software to adapt the dose applied to the skin. In order to correctly apply such software it is important to characterise the skin in terms of skin type with an easy and reliable method. 
Measuring the melanin content of the skin is the best method, but it needs to be done non-invasively. In a recent review Brenner and Hearing (7) discussed the photoprotective role and physiologic changes of melanin when human skin, covering the different Fitzpatrick Skin Types I to VI (8), is irradiated with ultra-violet (UV) light. Studies covered in the review where performed in vivo and/or in vitro and elucidated the melanosomal differences between the different skin types. Meinhardt et al (9) measured the absorption spectra of in vivo human skin from the lighter skin types before and after exposure to natural UV radiation, while Wan et al (10) measured the epidermal (including the Stratum corneum) transmittance of in vitro light and dark coloured human skin over the UVA, UVB and visible wavelength ranges. None of these studies however specifically determined epidermal melanin concentration in the different skin types. The absorption features of melanin is monotone in the visible and near infra-red parts of the spectrum (11), but over UV wavelengths melanin absorption display non-monotonic features (9) that may allow for more accurate ways of determining melanin concentration and therefore the expected absorption through the epidermal layer.

Human skin of the different Skin Types I to VI is not always readily available. Hence the use of skin-like phantoms (12). Previous results with skin-like phantoms representing Skin Types I to VI indicated the possibility of predicting some of the non-monotonic optical characteristics of real human skin, albeit in the visible wavelength range as opposed to the UV range in real human skin (13). The purpose of this study is to compare the experimentally measured absorption features of these liquid skin-like phantoms with computational simulated skin from the Realistic Skin Model (RSM) part of the Advanced Systems Analyses Program (ASAP®) software from Breault Research (14). This software package has been used by the authors (15) and others (16-18). This study forms the first phase of the larger objective of knowledge acquisition about the optical properties of real human skin cell tissue to enhance the accuracy of these computational simulated skin models. 


\section{MATERIALS AND METHODS}

Computer simulations. The ASAP® commercial software from Breault Research is fundamentally a flexible and efficient optical system raytracing modelling tool, allowing users to easily make geometry changes to a tissue model. The ray tracing is based on the Monte Carlo ray tracing techniques, where "photons" or "rays" are traced through an optical medium with pre-defined absorption and scattering properties. The rays can automatically split into reflected, refracted, diffracted, polarized and scattered components as they propagate through the system. Each of the rays proceeds independently, interacting with the encountered optical surfaces in any order, as appropriate. This type of ray tracing is often described as "non-sequential" ray tracing. It is a simulation, based on the way that real light waves behave in the real world.

The Realistic Skin Model (RSM) part of the ASAP® software from Breault Research, was used as the modeling tool for this work. The skin model consisted of four layers, the Stratum corneum (SC), the epidermis (containing the melanosomes, the main absorbing particles), the dermis and the hypodermis. The hypodermis was not taken into account in this study. A graphical layout of the model is shown in Figure 1. The optical properties for the different layers in the model are reported in Tables 1 and 2.

\section{$>$ Figure $1<$}

The RSM model parameters for the eumelanin and pheomelanin concentrations in the epidermis are $80 \mathrm{~g} / \mathrm{L}$ and $12 \mathrm{~g} / \mathrm{L}$ respectively. The eumelanin and pheomelanin concentrations were kept at the above values for all skin types, while the value for the volume 
fraction of the melanosomes was varied (Table 1). The software multiplies these melanin concentrations and the volume fractions to calculate the amount of melanin in the epidermal layer.

Different skin types were simulated by changing the volume fraction of the melanosomes in the epidermis (19) as indicated in Table 1. The variation used corresponds to published data that reported a more than 10 fold increase in the absorption coefficient (from $0.2 \mathrm{~mm}^{-1}$ to $\left.2.5 \mathrm{~mm}^{-1}\right)$ between Caucasian and African skin at $694 \mathrm{~nm}(20,21)$. In humans, the thickness of the epidermis varies from $38 \mu \mathrm{m}$ in the cheek to $369 \mu \mathrm{m}$ on the finger tip (22). Two different sun exposed skin areas were evaluated in this work, the cheek (epidermal thickness of $38 \mu \mathrm{m})$ and the back of the hand $(87.5 \mu \mathrm{m})$. A Gaussian laser beam (power $10 \mathrm{~mW}$ ) with a beam waist of $0.4 \mathrm{~mm}$ was used as the light source in the model. Wavelengths between 300 and $1000 \mathrm{~nm}$ were used in this work. The shorter wavelengths were used due to the absorption features of melanin in the UV part of the spectrum and the other wavelengths selected are wavelengths that are applicable in laser treatments. The four important optical parameters that influence the propagation of light through tissue are the absorption coefficient $\left(\mu_{a}\right)$, the scattering coefficient $\left(\mu_{s}\right)$, the anisotropy factor $(g)$ and the refractive index $(n)(23)$. For this study published data of the optical parameters were used $(19,22,24)$. The values of $\mu_{a}$ and $\mu_{s}$ are determined by the concentrations of the absorbing and scattering particles in the different skin layers. The thickness of the different layers $(d), g$ and $n$ are listed in Table 2. The surface of the model is $10 \mathrm{~mm}^{2}$ and the depth ( $\mathrm{Z}$ dimension) varies from 1.853 to $1.9025 \mathrm{~mm}$, depending on the epidermal thickness. The values of both $n$ and $g$ (2) were kept the same over the wavelength range. In the model the optical properties for each layer are specified separately, but assumed uniform throughout a specific layer. Each photon is tracked until it is either absorbed in the model or exits the boundaries of the model. The skin model was divided into 150 layers and each layer into 150x150 segments to form small 3- 
dimentional volumes or voxels. The photons absorbed in each of the voxels were registered to give the total absorption in each layer.

Liquid melanin skin-like phantoms: The sample preparation and absorbance measurements on the liquid melanin skin-like phantoms have been described in detail in Smit et al (13) and only the information necessary for comparison with the computer simulations is repeated here.

Liquid melanin skin-like phantoms: Sample preparation. Synthetic melanin samples at concentrations ranging from $0.011 \mathrm{mg} / \mathrm{ml}$ to $1.3 \mathrm{mg} / \mathrm{ml}$ were prepared from a synthetic eumelanin (Sigma-Aldrich M0418) stock solution at physiological $\mathrm{pH}(\sim 7.01)$ as previously described in Smit et al (13). Skin-like phantoms were prepared by adding either $3 \mu \mathrm{l}$ or $30 \mu \mathrm{l}$ Intralipid (IL) (Sigma-Aldrich I141) to $3 \mathrm{ml}$ of each of the melanin samples. Intralipid is a phospholipid-stabilized soybean oil, supplied as $20 \%$ fat emulsion.

Liquid melanin skin-like phantoms: Experimental sample characterisation. A UV-VIS spectrophotometer (Shimazdu UV-1650 PC) using standard $1 \mathrm{~cm}$ pathlength cuvettes over the wavelength range 370 to $900 \mathrm{~nm}$ was used to measure sample absorbance spectra. Total attenuation coefficients $\left(\mu_{t}(\lambda)\right)$ for the skin-like phantoms were calculated from these. The total attenuation coefficient $\left(\mu_{t}(\lambda)\right)$ is the decrease in the light intensity through the sample and is equal to the combination of the absorption and scattering coefficients of the sample (5). It was calculated from 


$$
\mu_{t}(\lambda)=\frac{-\ln T(\lambda)}{L} \quad[1 / \mathrm{cm}]
$$

with $\quad T(\lambda)$ the wavelength dependent transmittance and $L$ the optical pathlength (in $\mathrm{cm}$ ).

\section{RESULTS}

UV-VIS absorbance spectra over the wavelength range 370 to $900 \mathrm{~nm}$ of melanin only samples were measured before and after addition of the Intralipid (IL) as presented in Smit et al (13). The most relevant results are repeated here in Figure 2 to facilitate comparison with the computer simulation.

Absorbance curves (in Figs. 2(a) and (b)) were calculated by subtracting the absorption of melanin-free IL solutions (representing "vitiligo" skin) from the absorption of the liquid skin-like phantoms with the same IL concentration. A more detailed explanation can be found in Smit et al (13). The skin-like phantoms represented fair $(0.011 \mathrm{mg} / \mathrm{ml}$ melanin), medium $(0.066 \mathrm{mg} / \mathrm{ml}$ melanin $)$ and dark $(0.13 \mathrm{mg} / \mathrm{ml}$ melanin $)$ skin phototypes. Medium to dark phantoms displayed non-monotonic trends towards shorter wavelengths. The peaks became more pronounced and shifted towards longer wavelengths as the melanin concentration was increased.

\section{$>$ Figure $2<$}

Increasing the amount of added IL (representing a thicker epidermal layer) led to increased attenuation in fairer phantoms; which was not observable in darker phantoms. The non-monotonic peaks were also broadened compared to peaks from spectra representing a thinner epidermal layer (compare Fig. 2(b) to 2(a)). A more detailed discussion on the skinlike phantoms can be found in Smit et al (13). 


\section{$>$ Figure $3<$}

The absorption coefficient by two different thicknesses of modelled human skin was calculated from simulations performed with the Realistic Skin Model (RSM) part of the ASAP® software for a skin depth up to $0.12 \mathrm{~mm}$ (Figs. 3(a) and (b)). Values for the absorption coefficient falls within the range of values measured by Meinhardt et al (9). The different melanin concentrations for the different skin types as displayed in Fig. 3 are given in Table 1. These concentrations are higher than the concentrations used in the liquid skin-like phantoms in Fig. 2. The lower concentrations used in the latter was necessitated by the absorbance saturation limitations of the spectrophotometer used as explained in Smit et al (13). However, the scale ratios for the phantoms' melanin concentrations were chosen to coinside with the melanin concentrations as used for the different skin types in the RMS model. Spectra displayed similar non-monotonic trends towards shorter wavelengths as spectra of the measured skin-like phantoms in Fig. 2, although more pronounced for fairer skin types in the former compared to the latter. In the thicker epidermis $(\mathrm{ET}=87.5 \mu \mathrm{m})$, fairer skin types predicted higher absorption of incoming light relative to darker skin types compared to similar spectra for the skin-like phantoms. This was different for the results from the skin-like phantoms. Peaks also tended to shift towards longer wavelengths as modelled skin darkness increased, but were much broader than peaks for the skin-like phantoms.

\section{DISCUSSION}

In this study the absorption features of liquid skin-like phantoms were measured over the wavelength range 370 - $900 \mathrm{~nm}$ (UVA and VIS) and compared to modelled skin simulated with the RSM part of the ASAP® software. The skin-like phantoms contained different 
concentrations of melanin and either $3 \mu \mathrm{l}$ or $30 \mu \mathrm{IL}$ were added to the samples to represent thinner and thicker skin. Modelled skin represented fair, medium and dark skin types of two different epidermal thicknesses (ET $=38 \mu \mathrm{m}$ and $87.5 \mu \mathrm{m}$ respectively).

Skin type (phototype) is determined by a combination of melanin and haemoglobin, together with other pigments including bilirubin and carotene. Epidermal melanin can broadly be divided into two types, eumelanin (black-brown colour) and pheomelanin (yellowreddish colour) $(3,4)$. Haemoglobin dominates the absorption properties of the dermis $(25,26)$. Although different studies differ on the ratios of pheomelanin to eumelanin concentration between the different skin types, in general it is agreed that lighter skin types contains a higher concentration pheomelanin compared to eumelanin that the darker skin types $(27,28)$. The ratio of the default values of $80: 12$ for the eumelanin to pheomelanin epidermal concentrations in the RSM model seemed to better reflect ratios in the dark skin types than the fairer skin types. This could have contributed towards the overestimation in the calculated absorption for the fairer skin types.

The melanocytes containing the melanosomes responsible for the biosynthesis and storing of the melanin are located in the basal layer of the epidermis $(3,29)$. In reviews on skin of colour, Taylor (30) and Brenner and Hearing (7) referred to studies that established that the number of melanocytes is racial independent, i.e. skin type independent. It may differ between individuals of the same skin type and even from one anatomical region to the next in the same individual. Differences in skin type are dependent on variations in the size, number and aggregation of the melanosomes, both inside the melanocytes and keratinocytes, as well as on the distribution throughout the epidermis and Stratum corneum $(7,30$ and references therein). For instance, Toda et al (31) and Olson et al (32) demonstrated differences in melanosome aggregation and size between dark skinned and light skinned individuals. 
Eumelanin is associated with larger elliptical melanosomes, while pheomelanin is associated with smaller spherical melanosomes. Melanosomes are also more evenly distributed throughout the epidermis and Stratrum corneum in the darker skin types compared to fairer, unexposed skin types where the melanosomes are observed to cluster more in the basal part of the epidermis (30 and references therein). In the RSM software size and aggregation parameters are not taken into account; the melanosome contribution is only represented as a volume fraction. The lack of size and aggregation representation in the model could possibly lead to an over- or underestimation of the melanin concentration for a specific skin type. Furthermore, each of the skin layers in the model is assumed a homogeneous medium. The melanin would therefore be considered evenly distributed throughout the epidermis, which favours the representation of the darker skin types more compared to the fairer skin types. These differences may hence partially lead to a possible overestimation in absorption calculated for the fairer skin types compared to real human skin.

The addition of IL to the liquid melanin only samples resulted in skin-like phantoms that displayed the non-monotonic absorption features observed in real human skin, albeit more towards the visible than the observed UVA / UVB wavelength range in real skin (13). Compare the results from this study for example to Meinhardt et al (9) and Wan et al (10). The non-monotonic absorption features in the RSM model were similar to those of the skinlike phantoms. This confirmed that the observed spectral response was indicative of the melanin's immediate reaction to the absorbed light, since it is not possible with either the skin-like phantoms or the RSM model to model the long term effects of induced erythema as observed in the Meinhardt et al (9) and Wan et al (10) studies. Erythema induced by exposure to wavelengths in the UV region shows three distinct pigmentary responses, namely immediate pigment darkening (IPD), persistent pigment darkening (PPD) and delayed tanning response (DTR) (7). IPD occurs immediately after sun exposure and shows a broad 
peak in the UVA $(320-400 \mathrm{~nm})$ and visible wavelength regions $(33,34)$. PPD occurs a few hours after UV exposure and lasts a few days, while DTR appears 2-3 days after UV exposure, is induced by either UVA or UVB and lasts for about 3-4 weeks (7 and references therein). In the Meinhardt et al (9) study the subjects were re-evaluated after two weeks' sun exposure with absorbance spectra therefore exhibiting the characteristics of DTR with peaks in the UVB region. Toda et al (31), Olson et al (32) and Lavker and Kaidbey (35) also demonstrated differences in melanosome aggregation between sun exposed and non-exposed anatomical regions in the same individuals, highlighting the effect of the delayed response of sun exposure. The RSM model simulations and skin-like phantoms showed absorbance peaks in the UVA and visible regions and hence model the optical absorption of sunlight in skin and not any of the biological responses to sun exposure. The important effects of the delayed response after sun exposure could not be modelled with either the RSM model or skin-like phantoms, highlighting the need for a model and skin-like phantoms that can be used to study the effects of sun exposure.

In a previous paper we attributed the appearance of the non-monotonic features in the skin-like phantoms to the interaction between the melanin and the IL, which differed depending on the melanin concentration (13). IL is a phospholipid-stabilized soybean oil consisting of an emulsion of bilipid membrane fragments of various sizes, suspended in water. It is commonly used to mimic the scattering properties of human skin. Melanin is a family of polymorphous multifunctional biopolymers that readily binds to cations, anions, drugs and chemicals (3). Our previous results indicated that the melanin binds to the IL fragments, although we do not know the exact nature of this interaction. The melanin subcomplexes also tended to aggregate into increasingly larger particles as the concentration was increased and we argued the possibility that such larger aggregates may bind to lipid structures more readily than the smaller melanin particles associated with lower melanin 
concentrations. Our results may therefore be interpreted that "photo-protection in darker skin phototypes may not be only a function of epidermal melanin concentration, but that the interaction between the melanin complex and the lipid membranes found in the skin cells may play an equally important role" (13).

In conclusion results from both the RSM model and the liquid skin-like phantoms indicated that they can be used to study the absorbance of sunlight in skin and not the delayed effects of sun exposure in the UV and visible wavelength ranges. However, more comprehensive models and phantoms are necessary to model and predict the longer term effect of sun exposure for all skin types.

Acknowledgements - This work was supported by CSIR Parliamentary Grants. We thank Dr Raymond Sparrow and the Synthetic Biology ERA at CSIR Biosciences for use of their laboratory and equipment.

\section{REFERENCES}

1. Peng, Q., A. Juzeniene, J. Chen, L. O. Svaasand, T. Warloe, K. E. Giercksky, and J. Moan (2008) Lasers in medicine. Reports on Progress in Physics 71, 056701-1-05670128.

2. Wilson, B. C. and S. L. Jacques (1990) Optical reflectance and transmittance of tissues: Principles and applications. IEEE J. Quantum Electron. 26, 2186-2199.

3. Costin, G. E. and V. J. Hearing (2007) Human skin pigmentation: Melanocytes modulate skin color in response to stress. FASEB J. 21, 976-994. 
4. Liu, Y., L. Hong, K. Wakamatsu, S. Ito, B. Adhyaru, C. Y. Cheng, C. R. Bowers, and J. D. Simon (2005) Comparison of structural and chemical properties of black and red human hair melanosomes. Photochem. Photobiol. 81, 135-144.

5. Tuchin, V. V. (2007) Tissue Optics: Light Scattering Methods and Instruments for Medical Diagnostics. SPIE Press, Bellingham, Washington, USA.

6. Alaluf, S., S. Alaluf, A. Heath, N. I. K. Carter, D. Atkins, H. Mahalingam, K. Barrett, R. I. A. Kolb, and N. Smit (2001) Variation in melanin content and composition in type V and VI photoexposed and photoprotected human skin: The dominant role of DHI. Pigment Cell Res. 14, 337-347.

7. Brenner, M. and V. J. Hearing (2008) The protective role of melanin against UV damage in human skin. Photochem. Photobiol. 84, 539-549.

8. Fitzpatrick, T. B. (1988) The validity and practicality of sun-reactive skin types I through VI. Arch. Dermatol. 124, 869-871.

9. Meinhardt, M., R. Krebs, A. Anders, U. Heinrich, and H. Tronnier (2009) Absorption spectra of human skin in vivo in the ultraviolet wavelength range measured by optoacoustics. Photochem. Photobiol. 85, 70-77.

10. Wan, S., R. R. Anderson, and J. A. Parrish (1981) Analytical modeling for the optical properties of the skin with in vitro and in vivo applications. Photochem. Photobiol. 34, 493-499.

11. Dam, J. S. (2001) Optical analysis of biological media - continuous wave techniques. PhD Thesis, Lund Institute of Technology. 
12. Bashkatov, A. N., E. A. Genina, V. I. Kochubey, M. M. Stolnitz, T. A. Bashkatova, O. V. Novikova, A. Y. Peshkova, and V. V. Tuchin (2000) Optical properties of melanin in the skin and skin-like phantoms. In Proceedings of SPIE - The International Society for Optical Engineering, Vol. 4162, (Edited by V. V. Tuchin), pp. 219-226. SPIE - The International Society for Optical Engineering, Controlling Tissue Optical Properties: Applications in Clinical Study, Amsterdam, Netherlands, 4 July 2000.

13. Smit, J. E., A. F. Grobler, and R. W. Sparrow (2011) Influence of variation in eumelanin content on absorbance spectra of liquid skin-like phantoms. Photochem. Photobiol. 87, 64-71.

14. Breault Research (2011) Breault Research Organization - Optical Design and Analysis Software, Optical Engineering Services and Software Training. Available at: http://www.breault.com/. Accessed on 4 November 2011.

15. Karsten, A. E., A. Singh and M. W. Braun (2011) Experimental verification and validation of a computer model for light-tissue interaction. Lasers Med.Sci. (In Press, DOI: 10.1007/s10103-011-0926-x).

16. Mustafa, F. H., M. S. Jaafar, A. H. Ismail, and H. A. A. Houssein (2011) The effect of laser wavelength in photodynamic therapy and phototherapy for superficial skin diseases. In 2011 IEEE International Conference on Imaging Systems and Techniques, IST 2011 - Proceedings, pp. 232-236. 2011 IEEE International Conference on Imaging Systems and Techniques, IST 2011, Batu Ferringhi, Penang, Malaysia, 17 May 2011 18 May 2011.

17. Holcomb, P. S. (2006) Under the skin: Virtual prototyping with tissue models in "ASAP". Laser+Photonics 2006, 56-58. 
18. Stevenson, M. A. (2009) Human skin and tissue phantoms in optical software: Engineering design and future medical applications. BSc (Hons) Thesis, College of Optical Science, University of Arizona, USA.

19. Jacques, S. L. and D. J. McAuliffe (1991) The melanosome: threshold temperature for explosive vaporization and internal absorption coefficient during pulsed laser irradiation. Photochem. Photobiol. 53, 769-775.

20. Zhang, R., W. Verkruysse, B. Choi, J. A. Viator, B. Jung, L. O. Svaasand, G. Aguilar, and J. S. Nelson (2005) Determination of human skin optical properties from spectrophotometric measurements based on optimization by genetic algorithms. $J$. Biomed. Opt. 10, 1-11.

21. Zonios, G., J. Bykowski, and N. Kollias (2001) Skin melanin, hemoglobin, and light scattering properties can be quantitatively assessed in vivo using diffuse reflectance spectroscopy. J. Invest. Dermatol. 117, 1452-1457.

22. Whitton, J. T. and J. D. Everall (1973) The thickness of the epidermis. Brit. J. Dermatol. 89, 467-476.

23. Star, W. M. (1997) Light dosimetry in vivo. Phys. Med. Biol. 42, 763-787.

24. Van Gemert, M. J. C., S. L. Jacques, H. J. C. M. Sterenborg, and W. M. Star (1989) Skin optics. IEEE Trans. Biomed. Eng. 36, 1146-1154.

25. Salomatina, E., B. Jiang, J. Novak, and A. N. Yaroslavsky (2006) Optical properties of normal and cancerous human skin in the visible and near-infrared spectral range. $J$. Biomed. Opt. 11.

26. Young, A. R. (1997) Chromophores in human skin. Phys. Med. Biol. 42, 789-802. 
27. Alaluf, S., D. Atkins, K. Barrett, M. Blount, N. Carter, and A. Heath (2002) Ethnic variation in melanin content and composition in photoexposed and photoprotected human skin. Pigment Cell Res. 15, 112-118.

28. Ito, S. and K. Wakamatsu (2003) Quantitative analysis of eumelanin and pheomelanin in humans, mice, and other animals: A comparative review. Pigment Cell Res. 16, $523-$ 531.

29. Del Bino, S., J. Sok, E. Bessac, and F. Bernerd (2006) Relationship between skin response to ultraviolet exposure and skin color type. Pigment Cell Res. 19, 606-614.

30. Taylor, S. C. (2002) Skin of color: Biology, structure, function, and implications for dermatologic disease. J. Am. Acad. Dermatol. 46, S41-S62.

31. Toda, K., M. A. Pathak, J. A. Parrish, T. B. Fitzpatrick, and W. C. Quevedo Jr (1972) Alteration of racial differences in melanosome distribution in human epidermis after exposure to ultraviolet light. Nat. New. Biol. 236, 143-145.

32. Olson, R. L., J. Gaylor, and M. A. Everett (1973) Skin color, melanin, and erythema. Arch. Dermatol. 108, 541-544.

33. Irwin, C., A. Barnes, D. Veres, and K. Kaidbey (1993) An ultraviolet radiation action spectrum for immediate pigment darkening. Photochem. Photobiol. 57, 504-507.

34. Pathak, M. A., F. C. RILEY, and T. B. Fitzpatrick (1962) Melanogenesis in human skin following exposure to long-wave ultraviolet and visible light. J. Invest. Dermatol. 39, 435-443. 
35. Lavker, R. M. and K. H. Kaidbey (1982) Redistribution of melanosomal complexes within keratinocytes following UV-A irradiation: A possible mechanism for cutaneous darkening in man. Arch. Dermatol. Res. 272, 215-228. 


\section{TABLES}

Table 1 Volume fractions of melanosomes used to represent the different skin types (19) and an estimate of the corresponding Fitzpatrick skin type (8)

\begin{tabular}{|c|c|c|c|}
\hline Skin type & $\begin{array}{c}\text { Fitzpatrick skin } \\
\text { type (estimate) }\end{array}$ & $\begin{array}{c}\text { Melanosome volume } \\
\text { fraction (percentage } \\
\text { as compared to Dark } \\
\text { Skin type) }\end{array}$ & $\begin{array}{c}\text { Melanin } \\
\text { concentration } \\
(\mathbf{m g} / \mathbf{m l})\end{array}$ \\
\hline Very fair & I-II & $0.0255(8.4 \%)$ & 2.346 \\
\hline Medium & IV-V & $0.155(50.8 \%)$ & 14.26 \\
\hline Dark & VI & $0.305(100 \%)$ & 28.06 \\
\hline
\end{tabular}


Table 2 Thickness $(d)$ in mm, anisotropy $(g)$ and refractive index $(n)$ of the different skin layers used

\begin{tabular}{|c|c|c|c|}
\hline Skin layer & Anisotropy (g) & $\begin{array}{c}\text { Refractive index } \\
\text { (n) }\end{array}$ & $\begin{array}{c}\text { Thickness (d) } \\
(\mathbf{m m})\end{array}$ \\
\hline Stratum corneum & 0.9 & 1.53 & 0.015 \\
\hline Epidermis & 0.79 & 1.5 & $0.038,0.0875$ \\
\hline Dermis & 0.82 & 1.4 & 1.8 \\
\hline
\end{tabular}




\section{FIGURES}

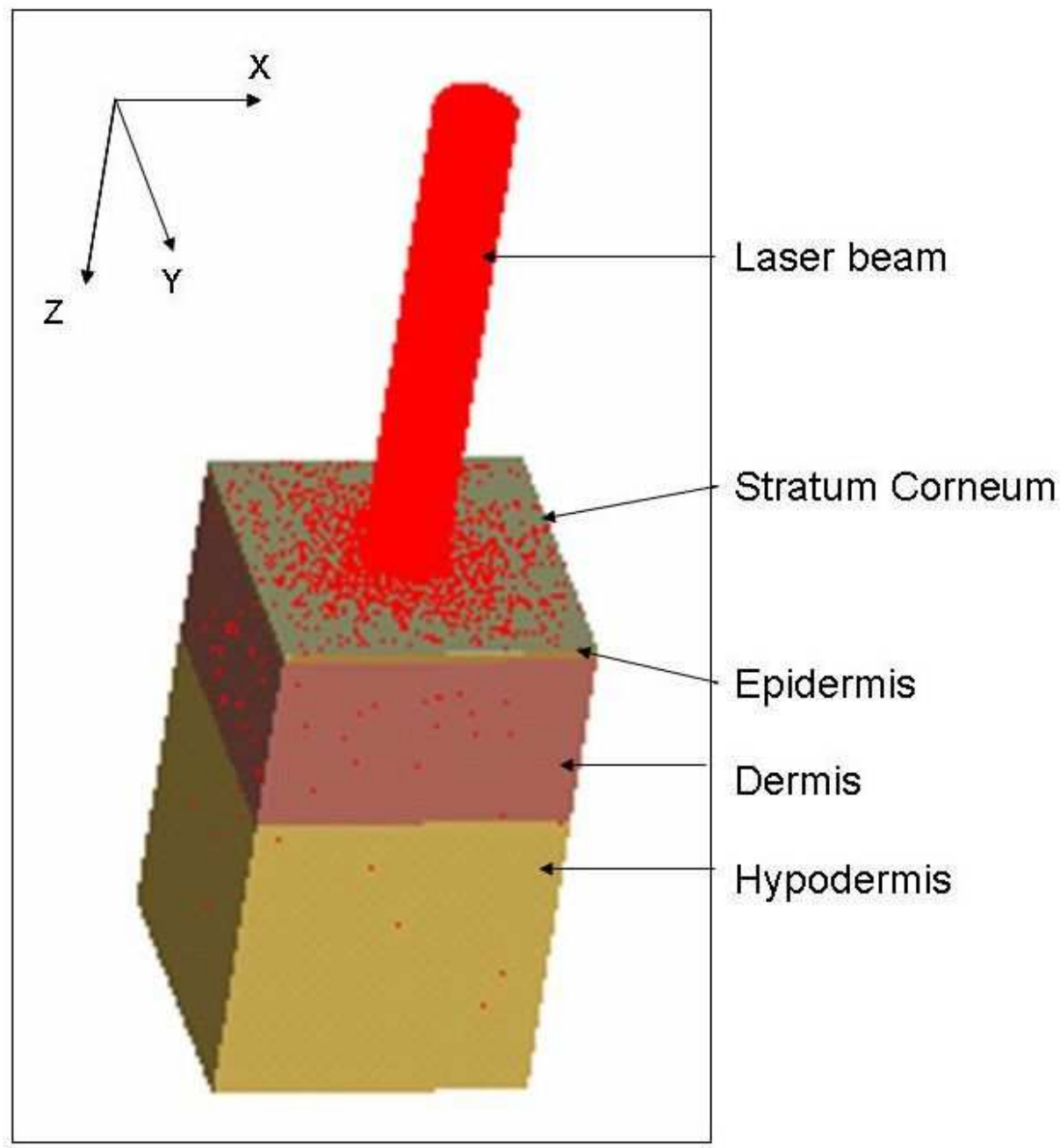

Figure 1. The Realistic Skin Model (RSM) showing the Stratum corneum, epidermal and dermal skin layers as well as the excitation laser beam (Gaussian profile and beam diameter of $0.4 \mathrm{~mm}$ ). The red spots on the model boundaries are the photons leaving the model. 

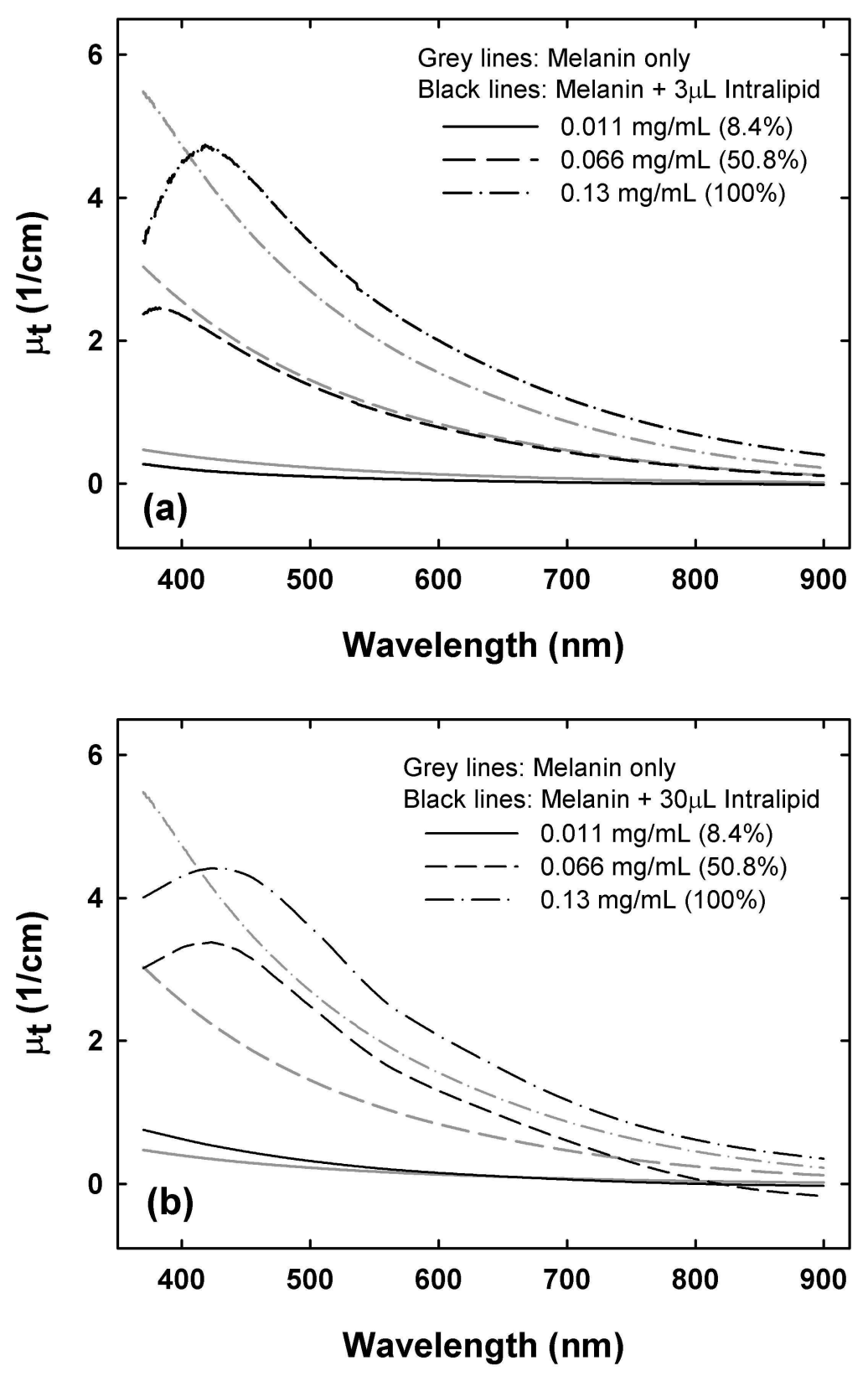

Figure 2. Comparison of absorbance spectra of skin-like phantoms containing (a) $3 \mu 1$ Intralipid and (b) $30 \mu 1$ Intralipid (black lines). Phantoms are also compared to the melanin only samples (grey lines) of same concentrations from Figure 2 in Smit et al (13). Melanin concentrations of $0.011 \mathrm{mg} / \mathrm{ml}$ (solid line), $0.066 \mathrm{mg} / \mathrm{ml}$ (dashed line) and $0.13 \mathrm{mg} / \mathrm{ml}$ (dotdashed line) were used to represent light, medium and dark skin types. Each concentration is also represented as a percentage compared to the highest concentration used (compare with Table 1). Adapted from Smit et al (13). 

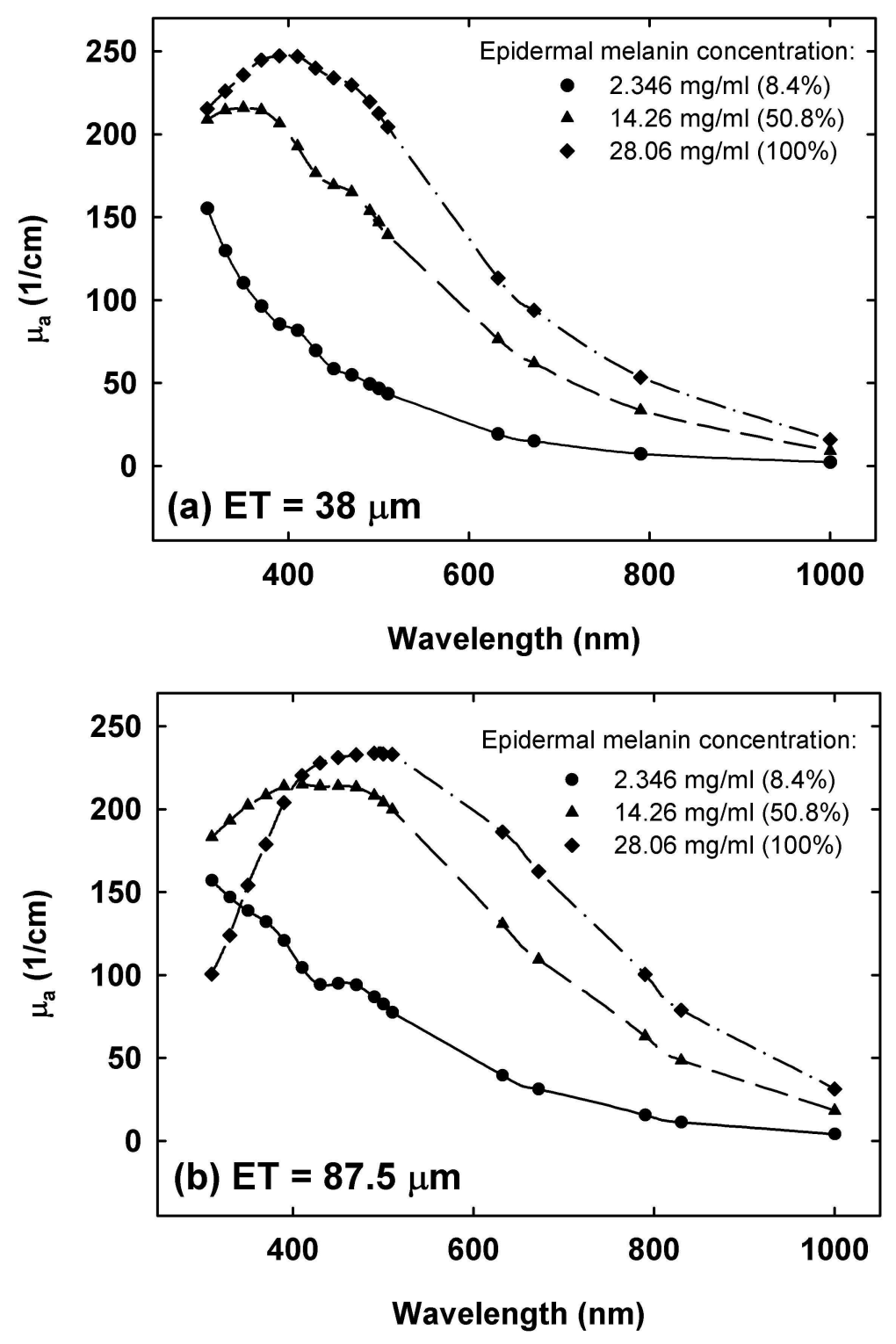

Figure 3. Comparison of the absorption coefficient as a function of wavelength for epidermal thicknesses (ET) of (a) $38 \mu \mathrm{m}$ and (b) $87.5 \mu \mathrm{m}$ up to a skin depth of $0.12 \mathrm{~mm}$ for epidermal melanin concentrations representing very fair, medium and dark skin types respectively. Each concentration is also represented as a percentage compared to the highest concentration used (compare with Table 1). 